\title{
Alleviating Memory Impairment through Distraction
}

\author{
Nathan Cashdollar, ${ }^{1,2,3}$ Nilli Lavie, ${ }^{2}$ and Emrah Düzel2,4,5 \\ ${ }^{1}$ Institute of Neurology and National Hospital for Neurology and Neurosurgery, University College London, London WC1N 3BG, United Kingdom, ${ }^{2}$ Institute \\ for Cognitive Neuroscience, University College London, London WC1N 3AR, United Kingdom, ${ }^{3}$ Center for Mind/Brain Sciences, University of Trento, \\ Trento, Italy 38060, ${ }^{4}$ Institute of Cognitive Neurology and Dementia Research, Otto-von-Guericke University, Magdeburg, Germany 39120 , and ${ }^{5} \mathrm{German}$ \\ Centre for Neurodegenerative Diseases, Magdeburg, Germany 39120
}

Distraction typically has a negative impact on memory for recent events and patients with existing memory impairment are particularly vulnerable to distractor interference. In contrast, here we establish a beneficial effect for distractor presentation in humans for both patients with memory impairment due to bilateral hippocampal lesions and healthy adults with low memory performance. Recognition memory for images of place scenes, which had to be memorized for short delay periods was significantly improved with the presentation of a distractor face during the delay. Magnetoencephalography recordings of neural oscillations in the theta frequency range obtained in healthy adults suggest that this memory improvement results from the interruption of rehearsal by the distractor. Our results highlight circumstances where active memory rehearsal may paradoxically increase memory impairments and distraction alleviates these memory deficits in patients with hippocampal injury and healthy adults.

\section{Introduction}

New memories are vulnerable to distraction. It is well established that this vulnerability makes distraction a major cause of memory impairment in amnesia (primarily due to damage to the hippocampus; Vargha-Khadem et al., 1997; Squire et al., 2004; Saksida and Bussey, 2010); and other clinical disorders such as stroke, epilepsy, and neurodegeneration; as well as in old age. The negative effects of distraction are particularly apparent in memory for events that occurred just "a moment ago," that is just before the distracting event (McGeoch and McDonald, 1931), and can become more susceptible to interference from distraction when the amount of information to be maintained (memory load) is increased (Chao and Knight, 1995; Baddeley, 1998; Chao and Knight, 1998; Baddeley, 2003).

During periods of short-term visual memory retention (i.e., rehearsal), the negative effects of distraction are most pronounced when the interfering stimuli are categorically related to those currently held in memory (Clapp et al., 2010). For instance, it has been demonstrated that when participants are required to maintain pictures of natural scenes over brief intervals, distracting pictures of faces during delay periods have little effect on performance (Clapp et al., 2010). However, when the distracting

Received April 30, 2013; revised Sept. 24, 2013; accepted 0ct. 23, 2013.

Author contributions: N.C., N.L., and E.D. designed research; N.C. performed research; N.C. analyzed data; N.C., N.L., and E.D. wrote the paper.

This work was supported by the Brain Research Trust and was undertaken at University College London Hospital/ University College London, which received a portion of funding from the Department of Health's National Institute for Health Research Biomedical Research Centre's funding scheme. Additional support came from the German Research Council (Grant KFG 163) and the Volkswagen Foundation. We thank Dr. Fergus J. Rugg-Gunn and Prof. John

S. Duncan for their valuable contribution of coordinating patient referrals and scheduling.

The authors declare no competing financial interests.

Correspondence should be addressed to Nathan Cashdollar, CIMeC-Università degli Studi di Trento, via delle Regole, 101, Mattarello, Trento, Italy 38060. E-mail: nate.cashdollar@gmail.com.

DOI:10.1523/JNEUROSCI.1797-13.2013

Copyright $\odot 2013$ the authors $\quad 0270-6474 / 13 / 3319012-11 \$ 15.00 / 0$ stimulus was congruent with the current memorandum (i.e., memory stimuli were faces and delay distractors were also faces) and the participant was required to make a cognitive judgment on the distractor stimuli (i.e., indicate if male or female face), memory performance decreased significantly (Clapp et al., 2010). Furthermore, this negative impact of task-related visual distraction has been found to be exacerbated in memory deficits associated with normal aging (Clapp and Gazzaley, 2012) and in patients with memory impairment after bilateral lesions of the hippocampus (Vargha-Khadem et al., 1997; Squire et al., 2004; Saksida and Bussey, 2010), a brain region frequently associated with amnesia (Vargha-Khadem et al., 1997; Squire et al., 2004).

Here we report a novel and clinically relevant exception to the negative effects of distraction. Using task-unrelated distractors, we discovered a beneficial effect of distractor presentation during short-term memory delays. We observed this benefit in patients with bilateral damage to the hippocampus who are typically impaired when short-term memory load is high (Shrager et al., 2008; Jeneson et al., 2011; Jeneson et al., 2012). Additionally, after matching memory performance in healthy participants to those of the patients by increasing the length of the delay, we demonstrate that distractor presentation can also improve memory performance in healthy adults. Neural synchrony within the theta-band $(4-8 \mathrm{~Hz})$ is a plausible physiological mechanism mediating active memory maintenance (Jones and Wilson, 2005; Fuentemilla et al., 2010), or working memory rehearsal (Fuentemilla et al., 2010), and previous investigations using magnetoencephalography (MEG) recordings have shown that increases in memory load enhance bifrontal synchrony in the theta frequency range (Cashdollar et al., 2009). Using this approach, we identify the physiological underpinnings of this beneficial effect of distractor presentation in healthy adults in whom frontal theta synchrony is interrupted by task-unrelated distraction during high memory load rehearsal in cases where distraction benefits performance. 
Table 1. Neuropsychological scores in BHS patients

\begin{tabular}{lccrrc}
\hline Subject & E.M. & M.R. & D.H. & G.P. & T.E. \\
\hline VIQ & 113 & 80 & 104 & 93 & 96 \\
Vocabulary & 10 & $*$ & 8 & 8 & 6 \\
Similarities & 10 & $*$ & 7 & 7 & 9 \\
Arithmetic & 11 & $*$ & 10 & 10 & 11 \\
Digit span & 12 & 5 & 11 & 11 & 10 \\
PC & 11 & $*$ & 10 & 10 & $*$ \\
Verbal recall (immediate) & 39 & $*$ & 12 & 22 & $*$ \\
Verbal recall (delayed) & 31 & $*$ & 0 & 0 & $*$ \\
Verbal learning 1-5 (immediate) & 50 & $*$ & 31 & 37 & 40 \\
Verbal learning 1-5 (delayed) & 11 & $*$ & 5 & 3 & 4 \\
Figure recall (immediate) & 57 & $*$ & 59 & 26 & $*$ \\
Figure recall (delayed) & 37 & $*$ & 0 & 36 & $*$ \\
Design learning 1-5 (immediate) & 24 & $*$ & 28 & $*$ & $*$ \\
Design learning 1-5 (delayed) & 5 & $*$ & 5 & $*$ & $*$ \\
McKenna naming & 18 & $*$ & $*$ & 22 & 16 \\
MRI & 1.5 T & 1.5 T & 3 T & $*$ & 3 T \\
Volume right & 2.47 & 2.01 & 1.44 & $*$ & 1.42 \\
Volume left & 1.8 & 1.76 & 0.95 & $*$ & 1.04 \\
\hline
\end{tabular}

Raw neuropsychological scores and hippocampal volumes for patients with BHS. *Please note that some scores were not available.

\section{Materials and Methods}

Study 1

Patient groups. Five right-handed patients with temporal lobe epilepsy and bilateral hippocampal sclerosis (BHS; 2 females, mean age $43.2 \pm$ 10.9 years) participated in the experiment as the testing group (same cohort as reported previously in Cashdollar et al., 2009; see Table 1 for neuropsychological assessment scores). Six right-handed patients with temporal lobe epilepsy (TLE; 1 female, $36.7 \pm 12.4$ years) determined to be MRI negative for hippocampal volume reductions and signal abnormalities served as a control group to match the effects of recurrent epileptic seizures and antiepileptic medication in BHS patients, but without hippocampal sclerosis. The TLE group was well balanced, with 3 left and 3 right hemisphere dominant TLE patients. All patients underwent comprehensive clinical whole-brain MRI scans including the following: T1-weighted, proton density, T2-weighted, and FLAIR acquisition protocols. All patients in this study were inspected with special epilepsyfocused imaging protocols highly suitable to visually detect signal abnormalities inside and outside of the hippocampus. Furthermore, the interpretation of MR images and neuropsychological assessments involved a comprehensive inspection by epileptologists, neuroradiologists, and radiographers experienced in the presurgical evaluation of patients with epilepsy of this kind. Using this strict diagnostic procedure, only patients with no structural abnormalities (this includes the entorhinal and perirhinal cortices) other than bilateral hippocampal sclerosis were included in the BHS group. Also, no structural abnormalities were determined to be found in the TLE patients (Fig. 1 provides sample T1weighted images showing isolated bilateral hippocampal atrophy in BHS and normal-appearing hippocampi in the MRI-negative TLE cohort). All patients were attending clinics at the Department of Clinical and Experimental Epilepsy of the University College London (UCL) Institute of Neurology and the National Hospital for Neurology and Neurosurgery. All patients gave written informed consent and the study was approved by the UCL Research Ethics Committee for Human-Based Research.

Healthy participants. Fifteen normal controls (NC1; 9 females, mean age $24.7 \pm 3.9$ years) participated in the same experimental paradigm. Participants in Studies 1 and 2 possessed an undergraduate/graduate degree or were currently enrolled in undergraduate/graduate level degree courses (NC1 and NC3). Magnetoencephalography recordings were collected on 10 of these participants while they were undergoing the experimental procedure. Participants for these experiments gave written informed consent and the study was approved by the UCL Research Ethics Committee for Human-Based Research. Healthy participants for both Studies 1 and 2 were compensated at a rate of $\mathfrak{E} 6 / \mathrm{h}$ for their time. In addition, a second group of 13 normal controls (NC2) were recruited by
A

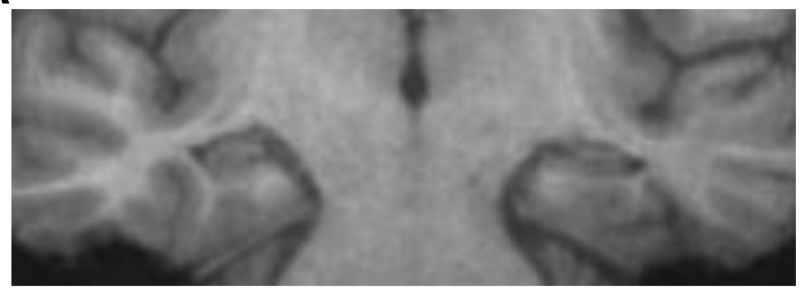

B

Patient (BHS): TE 015

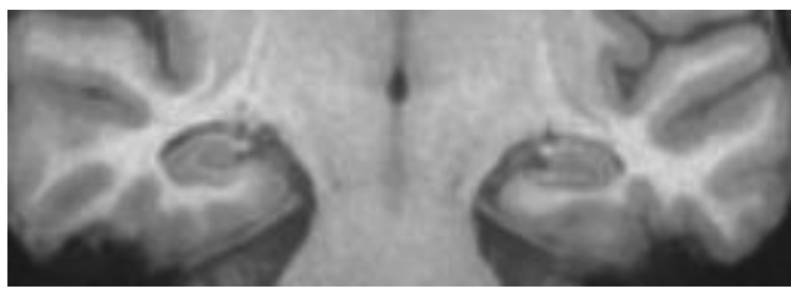

Patient (TLE): FT 026

Figure 1. Sample T1-weighted images of epilepsy cohorts. A, Epilepsy patient TE015 with isolated BHS and no other apparent structural or signal abnormalities. B, Patient FT026 with temporal lobe epilepsy determined to be MRI negative for hippocampal volume reductions and signal abnormalities (TLE).

the Otto-von-Guericke University of Magdeburg that were age matched (7 females, mean age $46.6 \pm 6.9$ years) and education matched (NC2: 10th grade in Germany equivalent to BHS: GCSE standard in England, both secondary school level up to ages 16-17 years) with the BHS patients and tested post hoc to insure that any impairments in the BHS group were not due to age or educational differences. All NC2 participants gave written informed consent and the study was approved by the Ethics Committee of the Otto-von-Guericke University of Magdeburg for Human-Based Research. Participants were compensated at a rate of 8 $€ / h$ for their time.

Stimuli and task design. The stimuli and task design were as described previously (Cashdollar et al., 2009). Sample, foil, and probe stimuli consisted of 742 gray scale photographs of indoor or outdoor scenes. Distractor stimuli consisted of 105 gray scale photographs of male and female faces with neutral emotional expression selected from the Karolinska Directed Emotional Faces database. Presentation of indoor/outdoor and male/female stimuli were counterbalanced across each block and were kept constant across individual trials. All pictures were gray scaled and normalized to a mean gray value of 127 and an SD of 75 set at $300 \times 300$ pixels and shown upon a gray background (127 value).

The experiment was a $2 \times 3$ factorial design consisting of 7 successive delayed-match-to-sample blocks with 30 trials per block, resulting in 35 trials per condition. The manipulations in this experiment were sample stimulus load of 1,3 , or 5 items presented serially for $1 \mathrm{~s}$ duration each. On half of the trials, a face distractor stimulus $(1 \mathrm{~s})$ was presented during the delay period (jittered within a $3 \mathrm{~s}$ window around the middle of the delay).

Participants were instructed to maintain the sample stimuli $(1,3$, or 5 items presented serially) over a $5 \mathrm{~s}$ delay period while fixating on a cross and to ignore the face distractor stimuli. During this delay period, participants were asked not to move or blink their eyes. At probe, subjects were presented with a single picture $(1 \mathrm{~s})$ and asked to indicate by button press using the index or middle finger of the right hand if the picture was a "match" or "non-match" to one of the aforementioned sample stimuli. Targets and foils were randomized and counterbalanced across testing blocks, after which time, there was a $3.5 \mathrm{~s}$ intertrial interval in which subjects were instructed to blink before fixing on the next cue (0.5 s; Fig. 2).

$M E G$ recording and wavelet transformation. MEG data were recorded using a 275-channel CTF Omega whole head gradiometer system (VSM MedTech) at a $480 \mathrm{~Hz}$ sampling rate and $120 \mathrm{~Hz}$ low-pass filtering. After 

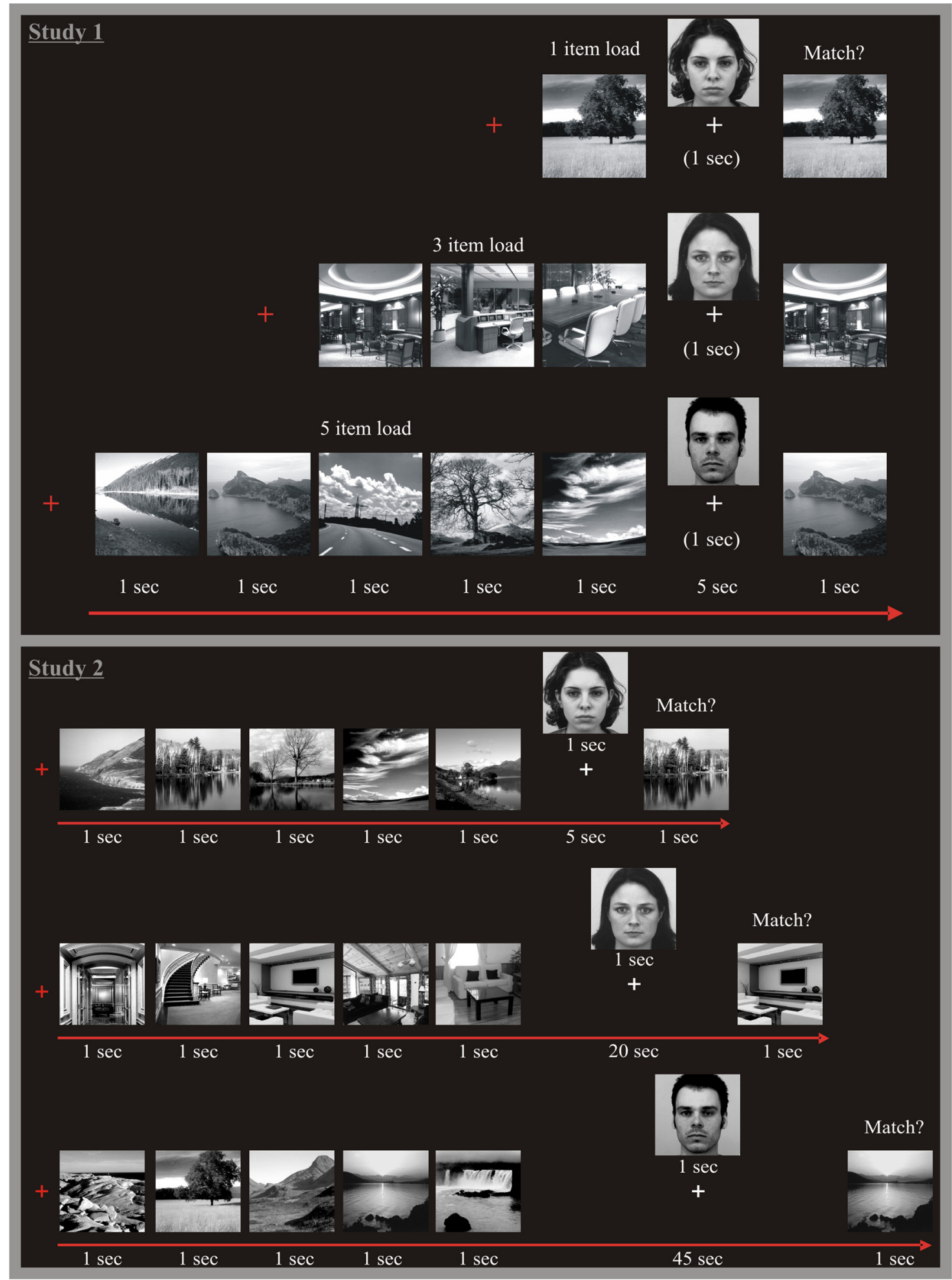

Figure 2. Delayed match to sample (DMS) memory recognition tasks. Study 1 (top): An example of trials in which 1, 3, or 5 items are presented serially and must be maintained over a $5 \mathrm{~s}$ delay period in order to make a "match/no match" decision at test. Study 2 (bottom): An example of trials in which 5 items are presented serially and must be maintained over a 5,20 , or $45 \mathrm{~s} \mathrm{delay} \mathrm{period}$ to make a "match/no match" decision at test. In both Study 1 and Study 2, a face distractor was presented for $1 \mathrm{~s}$ with a temporal jitter in the middle of the delay on $50 \%$ of trials.

participants were comfortably seated in the MEG, head localizer coils were attached to the nasion and preauricularly $1 \mathrm{~cm}$ anterior to the left and right tragus to monitor head movement during the recording sessions.

MEG data for the phase-coupling analysis were preprocessed with Statistical Parametric Mapping software implemented in MATLAB
(SPM5; Wellcome Trust Centre for Neuroimaging, UCL Institute of Neurology, London). Data were band-pass filtered to $0.5-100 \mathrm{~Hz}$ (Butterworth filtered) and the sampling rate was reduced to $140 \mathrm{~Hz}$. Data were epoched and then analyzed using continuous single-trial wavelet transformations within the theta band frequency ( 15 cycle Morlet wavelet with logarithmic scaling between 3 and $8 \mathrm{~Hz}$ ) using a MATLAB-based 
in-house software (Guderian and Düzel, 2005; Cashdollar et al., 2009). Single-trial transformations were analyzed separately for amplitude and phase coupling for each subject in the experiment.

Phase-coupling analysis. Based on previous results (Cashdollar et al., 2009; Fuentemilla et al., 2010), we selected, a priori, a $6 \mathrm{~Hz}$ wavelet (theta frequency) to measure functional coupling between sensor groups. Then using continuous single-trial wavelet transformations on unaveraged data (band-pass filtered between 3 and $9 \mathrm{~Hz}$, sampling every fourth time point), phase differences were calculated before averaging for each time point of each trial, between all possible sensor combinations of unique sensor pairs, and then averaged across trials. Phase alignment for each time point and sensor pair was measured as the length of the unit phase vector across trials divided by the number of trials. This computation yielded a complex value of phase synchronization ranging from 0 to 1 (Lachaux et al., 1999). A value of 1 would correspond to perfect phase alignment across trials and a value of 0 to random phase variation across trials.

Synchrony plots were then generated on these transformations, contrasting differences in sensor coupling between testing conditions after a $500 \mathrm{~ms}$ poststimulus baseline correction (threshold of $p<0.05$ ). Using these plots, we performed an unbiased search for theta coupling (for all possible combinations of MEG sensors) lasting at least three successive theta cycles. Sensor groups displaying significant theta synchrony and clustered with at least three other neighboring significant sensors were chosen for further region of interest analysis using serial related measures $t$ tests (threshold of $p<0.05$ ) to see spatial-temporal changes of theta coupling as a function of the experimental manipulation (Cashdollar et al., 2009).

\section{Study 2}

Healthy participants. A total of 21 healthy subjects ( 13 females, mean age $23.9 \pm 5.5$ years $)$ participated in this study ( 1 participant was excluded from the study due to not following task instructions). Recruitment, consent, and ethics approval were as in Study 1.

Stimuli and task design. The photographic stimuli and visual presentation settings are identical to that of the 5-item load condition of Study 1. This experiment was a $2 \times 3$ factorial design consisting of 4 successive blocks with 30 trials per block, resulting in 20 trials per condition. The manipulations in this experiment were delay lengths of 5,20 , or $45 \mathrm{~s}$. In half of the trials, a face distractor stimuli ( $1 \mathrm{~s}$ ) was presented during the middle of the delay period. Subjects were instructed to maintain the 5 sample stimuli over a variable delay period while fixating on a cross and to ignore the face distractor stimuli (Fig. 2). It is important to note that participants could not predict the delay length at the onset of a given trial or whether a distractor would be presented during the delay. Targets, foils, delay length, and distractor presentation were randomized and counterbalanced across testing blocks and participants.

\section{Results}

\section{Alleviating memory impairment in BHS}

In Study 1, five epilepsy patients with BHS (Fig. 1A) participated in a short-term memory recognition task. They were presented with a series of pictures of natural scenes and asked to keep these pictures actively in their mind. The number of scene items determined memory load to be low ( 1 item), moderate ( 3 items), or high ( 5 items). After a $5 \mathrm{~s}$ delay, a picture was presented and patients were asked to indicate whether the picture was part of the memory set or if it was entirely new. In half of the trials, a distractor face picture was presented ( $1 \mathrm{~s}$ ) during the memory delay (Fig. 2 ). BHS performance was compared to 15 healthy young university students (NC1) whose behavioral data were collected in conjunction with MEG recordings. A second control group (NC2) of 13 healthy adults was age and education matched to the BHS patients. In addition, six patients with TLE determined to be MRI negative for hippocampal lesions were tested to control for the effects of recurrent epileptic seizures and antiepileptic medication in BHS patients (Fig. 1B).
A $2 \times 3$ repeated-measures ANOVA with load (1, 3, 5 items) and distraction (distractor, no distractor) as the within-subjects factors and group (BHS, NC1) as the between-subjects factor revealed main effects for load $\left(F_{(2,36)}=45.84 ; p=0.0001\right)$, for distraction $\left(F_{(1,18)}=7.19 ; p=0.015\right)$, and for group $\left(F_{(1,18)}=\right.$ $16.95 ; p=0.001)$. There was a load-by-group interaction $\left(F_{(1.8,33.1)}=4.25 ; p=0.025,{ }^{*}\right.$ Greenhouse-Geisser corrected for violations of sphericity) indicating that the effects of load were greater for the BHS versus NC1s. Importantly, there was also an interaction of load, distractor presence, and group $\left(F_{(1.37,24.6)}=\right.$ 3.98; $p=0.046,{ }^{\star}$ Greenhouse-Geisser corrected for violations of sphericity) demonstrating a selectivity for the effects of distraction with increasing memory load to the BHS patients only (Fig. 3A).

A one-way ANOVA confirmed that, relative to control participants (NC1), BHS patients' recognition memory was markedly impaired in the higher item-load conditions (3-item: BHS $67.8 \pm$ $12.7 \%$ vs control $91.0 \pm 6.0 \%, F_{(1,18)}=31.55, p=0.0001 ; 5$-item: BHS $70.0 \pm 7.6 \%$ vs control $81.0 \pm 7.5 \%, F_{(1,18)}=7.87 p=$ 0.012 ), and to a lesser extent, the 1-item load condition (1-item: BHS $90.8 \pm 3.11 \%$ vs control $97.0 \pm 4.26 \%, F_{(1,18)}=8.70, p=$ $0.008)$. Distractor present conditions did not affect memory performance for controls (all ps $>0.11$; Fig. $3 A$ ), as expected given previous reports (Postle et al., 2005; Clapp et al., 2010) that distractors that are categorically unrelated to the memorandum have little or no effect on normal performance (Clapp et al., 2010). However, distractor presentation enhanced recognition memory for the BHS patients (Fig. $3 A$ ) such that differences to $\mathrm{NC} 1$ were attenuated to being nonsignificant in the 3 -item load and 5-item load conditions (all ps $>0.06$; Fig. $3 A$ ). The interaction of load, distractor presence, and group confirmed the selectivity of this distractor enhancement effect with increasing memory load to the BHS patients only. Therefore, distractor presentation elevated the memory performance of BHS patients to the level of a significantly younger (controls 24.7 years vs BHS 43.2 years) and relatively better educated group of healthy controls (NC1; please see Materials and Methods).

A follow-up ANOVA within the BHS patient group $(2 \times 3$ repeated-measures with distraction [distractor, no distractor] and load $[1,3,5$ items $])$ revealed main effects for load $\left(F_{(2,8)}=\right.$ 20.18; $p=0.001)$, distraction $\left(F_{(1,4)}=9.12 ; p=0.039\right)$, and a load-by-distraction interaction $\left(F_{(2,8)}=5.47 ; p=0.032\right)$, demonstrating a greater benefit of distraction at higher loads (Fig. $3 C$ ). Post hoc paired $t$ tests (2-tailed) within the BHS group demonstrated a significant increase in performance for the 3 -item distractor present condition $(81.2 \pm 3.4 \%)$ compared with no distraction $\left(67.8 \pm 5.7 \%, t_{(4)}=-3.55, p=0.024\right)$. Distractorrelated performance increases did not reach significance for 1 item (no distraction $90.8 \pm 1.4 \%$; with distraction $91.4 \pm 1.7 \%$, $t_{(4)}=-0.44, p=0.68$ ) or 5 items (no distraction $70.0 \pm 3.4 \%$; with distraction $\left.75.2 \pm 4.9 \%, t_{(4)}=-1.46, p=0.22\right)$. Thus, while BHS patients appear to improve in the higher load conditions when a distractor is present, this improvement is most reliable under moderate levels of load and less reliable when load is high (also see individual scores in Table 2 demonstrating that all BHS patients had increased accuracy for the higher load conditions with distraction; the only exception was patient T.E., who showed increased accuracy with distraction for only the 3-item condition).

To ensure that the BHS patients' memory impairments relative to NC1 were not due to group differences in age and education, we tested an alternate group of age- and education-matched healthy controls (NC2) on the same experimental procedure. 
Follow-up $2 \times 3$ repeated-measures ANOVAs comparing the BHS and NC2 groups revealed main effects for load $\left(F_{(2,32)}=39.4 ; p=0.0001\right)$, for distraction $\left(F_{(1,16)}=11.8 ; p=0.003\right)$, and for group $\left(F_{(1,16)}=10.1 ; p=0.006\right)$. There was a load-by-group interaction $\left(F_{(2,32)}=\right.$ 4.6; $p=0.018)$ indicating that the effects of load were again greater for the BHS versus NC2, as well as a distraction-by-group interaction $\left(F_{(1,16)}=4.93 ; p=0.041\right)$ demonstrating that distractor-related improvement was specific to the BHS group. There was also a significant load by distractionby-group interaction $\left(F_{(2,32)}=3.76\right.$; $p=$ $0.034)$, indicating that distractor-related improvements in the BHS group were more pronounced at loads of $>1$ item. These results mirror the results of the BHS comparison with the first normal control group ( $\mathrm{NC1}$ ). As in the comparison to the NC1 (younger and better educated) control group, one-way ANOVA showed that BHS patients were significantly impaired compared with NC2 for 3-item load conditions (BHS $67.8 \pm 5.7 \%$ vs NC2 $89.0 \pm$ $\left.1.6 \%, F_{(1,16)}=24.57 ; p=0.0001\right)$ and 5 -item conditions (BHS $70.0 \pm 3.4 \%$ vs NC2 $84.3 \pm 2.4 \%, F_{(1,16)}=10.50 ; p=$ $0.005)$. Therefore, the difference in age and education cannot account for the impairments found in BHS patients compared with the first control group and are also in agreement with previous reports of visual memory load impairments found in patients with hippocampal damage (Shrager et al., 2008; Jeneson et al., 2011; Jeneson et al., 2012). In addition, the increased performance in the BHS group on distractor present trials diminished these group differences at higher loads (3-item: BHS $81.2 \pm 3.4 \%$ vs NC2 $89.1 \pm 3.2 \%$, $F_{(1,16)}=1.97 ; p=0.179 ; 5$-item: BHS $75.2 \pm 4.9 \%$ vs NC2 $85.7 \pm 3.1 \%, F_{(1,16)}$ $=3.17 ; p=0.094$; Fig. $3 B$ ). The results of this comparison resemble that of $\mathrm{NC1}$ (Fig. 3A) and therefore rule out confounding influences of age or education level on the group comparisons.

In addition, six patients with TLE determined to be MRI negative for hippocampal lesions (Fig. $1 B$ ) were tested to control for the effects of recurrent epileptic seizures and antiepileptic medication in BHS patients. A $2 \times 3$ repeatedmeasures ANOVA with load (1, 3, and 5 items) and distraction (distractor, no distractor) within this TLE patient group showed a comparable main effect for load $\left(F_{(2,10)}=\right.$ $11.19 ; p=0.003)$ to that of the BHS patients, but no main effect for distraction $\left(F_{(1,5)}=0.097 ; p=0.77\right)$ or load-by-distraction interaction $\left(F_{(2,10)}=0.001 ; p=0.99\right)$. The pattern of results for the TLE patient group was also similar to both normal control groups (NC1 and NC2). Like the normal controls, the TLE pa-
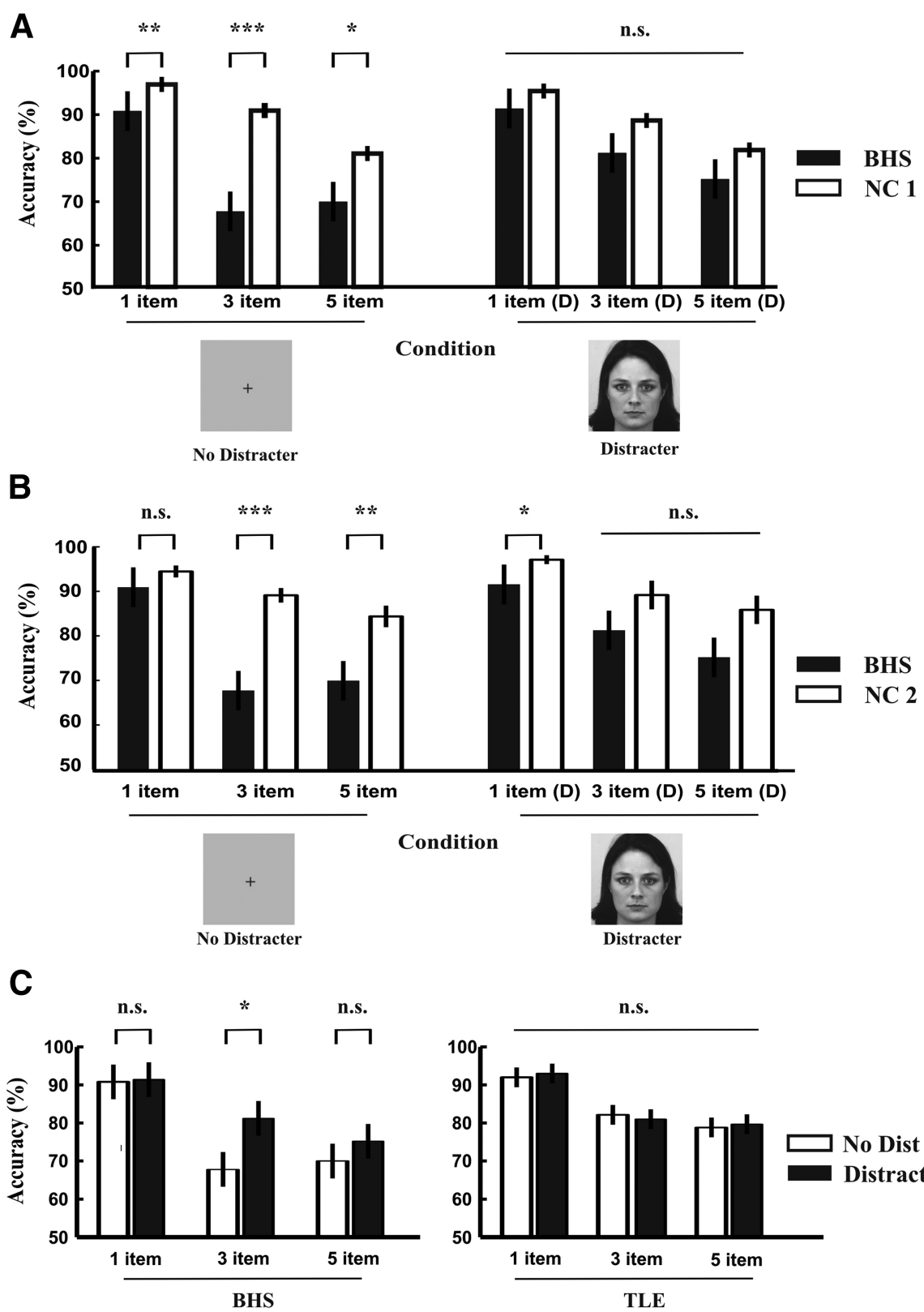

Figure 3. Delayed match to sample (DMS) memory load accuracy in Study 1. A, Comparisons of patients with BHS with NC1 normal control participants as a function of DMS memory load (1,3, or 5 items) and distractor presence (D). When no distractor was present during the delay period (left), BHS patients' performance was significantly reduced compared with NC1; however, when a face distractor was presented for $1 \mathrm{~s}$ during the delay period (right), performance in the high-load conditions was enhanced such that differences to NC1 were attenuated to being nonsignificant. Comparable to the NC1 (younger and better educated) control group (B), BHS patients demonstrate similar memory load impairments for higher DMS load (3 and 5 items) conditions without distraction (left) compared with a group of age- and education-matched normal controls (NC2). These group differences are again alleviated to be nonsignificant when distraction was presented during the retention period (right) for the higher DMS load ( 3 and 5 items) conditions. C, Mean accuracy for DMS tasks for BHS patients (left) and patients with TLE but no hippocampal damage (right). When distraction was presented during the retention period (black), BHS patients' memory performance increased compared to when no distractor was presented (white). No such performance differences were found in the TLE group between distractor present and distractor absent conditions. Error bars indicate SEM. ${ }^{*} p<0.05 ;{ }^{* *} p<0.01{ }^{* * *} p<0.001$.

tients were unaffected by distractor presentation in any condition of load (all follow-up paired $t$ tests confirmed that accuracy did not increase in distractor present conditions in the TLE group; all ps $>0.60$; Fig. $3 C$ ), confirming that the effects of distractor presentation in the BHS group cannot be explained in terms of disease status or medication. Instead, the findings clearly indicate that memory impairments, particularly under moderate levels of 
Table 2. Raw accuracy for BHS patients in Study 1

\begin{tabular}{lllllll}
\hline Subject & 1 item & 1 item (D) & 3 items & 3 items (D) & 5 items & 5 items (D) \\
\hline E.M. & $94 \%$ & $97 \%$ & $81 \%$ & $94 \%$ & $76 \%$ & $91 \%$ \\
M.R. & $94 \%$ & $91 \%$ & $71 \%$ & $78 \%$ & $60 \%$ & $68 \%$ \\
G.P. & $90 \%$ & $88 \%$ & $78 \%$ & $82 \%$ & $79 \%$ & $80 \%$ \\
T.E. & $87 \%$ & $88 \%$ & $56 \%$ & $74 \%$ & $69 \%$ & $63 \%$ \\
D.H. & $89 \%$ & $93 \%$ & $53 \%$ & $78 \%$ & $66 \%$ & $74 \%$ \\
\hline
\end{tabular}

All BHS patients demonstrated increases in accuracy for the higher load ( 3 item and 5 item) distraction conditions (D) compared to when no distractor was presented. The only exception is patient TE who showed increased accuracy for only the 3 item (D) condition.
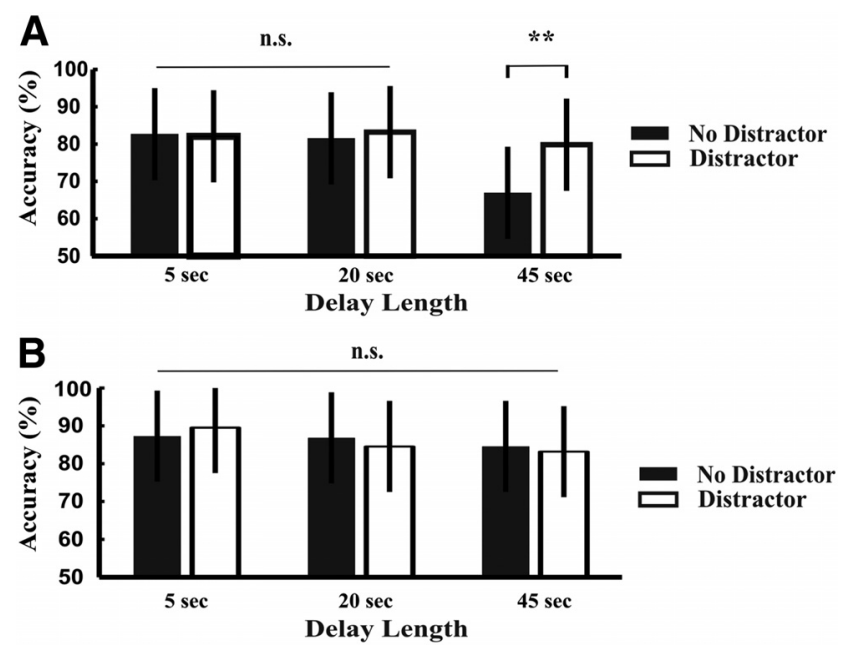

Figure 4. Study 2 investigated an additional group of normal controls (NC3) under high-load conditions (5 items) with extended memory delay durations $(5,20$, or $45 \mathrm{~s})$ to achieve similar levels of reduced memory performance as the BHS patients. A median split of the $45 \mathrm{~s}$ delay length condition without distraction (median value $=80 \%$ ) resulted in two groups: a low performance group matched for accuracy to that of the BHS patients $(\boldsymbol{A})$ and a high performance group $(\boldsymbol{B})$. $\boldsymbol{A}$, When the accuracy of the NC3 low performance group approached that of BHS patients for the high-load conditions $(<80 \%)$, distractor presentation during the retention interval significantly improved memory performance. $\boldsymbol{B}$, In the high performance group, no memory improvement was found with delay distraction. Error bars indicate SEM. ${ }^{* *} p<0.01$.

load, were selective to the BHS patients and that it was this condition-specific impairment that was alleviated by distractor presentation in this group.

\section{Alleviating low memory performance in normal controls}

In Study 2, we tested an additional group $(n=20)$ of normal controls (NC3) under the high-load condition (5 items) with extended memory delay durations $(5,20$, or 45 s; Fig. 2$)$ to achieve similar levels of reduced memory performance as the BHS patients (Materials and Methods). To better match performance of the NC3 group without distraction to that of the BHS patients, we performed a median split of the $45 \mathrm{~s}$ delay length condition with no distraction (median value $=80 \%$ accuracy), resulting in two groups: low performers $(<80 \%$; $n=9$ participants, cumulative $45 \%$, mean age $25.78 \pm 5.09$ years; 5 females) and high performers ( $\geq 80 \%, 11$ participants, cumulative $55 \%$, mean age $22.55 \pm 5.80$ years; 8 females). It is important to note that BHS patients' memory improvements with distraction were most apparent when mean performance fell below $80 \%$ (in the 3 -item and 5-item load conditions) and the mean accuracy of the low performance group $(66.1 \pm 2.9 \%)$ was now closely matched to that of the BHS patients (3-item load: $67.8 \pm 12.7 \%$; 5-item load: $70.0 \pm 7.6 \%$; Figs. $3,4 A$ ).

A $2 \times 3 \times 2$ repeated-measures ANOVA with delay length (5, 20, 45 s) and distraction (with and without) as the within- subjects factors and group (high and low performers) as the between-subjects factor revealed a main effect for delay $\left(F_{(2,36)}=\right.$ 7.08; $p=0.003)$, demonstrating that prolonged memory delays negatively affected performance. Importantly, there was also a distraction-by-group interaction $\left(F_{(1,18)}=5.00 ; p=0.038\right)$ showing that low performers were selectively affected by distractor-related performance improvements and a delay by distraction-by-group interaction $\left(F_{(2,36)}=4.08\right.$; $\left.p=0.025\right)$, indicating that, similar to BHS patients, these condition-specific performance deficits are what is alleviated in the low performers.

Follow-up $2 \times 3$ repeated-measures ANOVA with delay length $(5,20,45 \mathrm{~s})$ and distraction (with and without) as the within-subjects factors for the low performance group demonstrated main effects for delay $\left(F_{(2,16)}=7.83 ; p=0.004\right)$ and distraction $\left(F_{(1,8)}=10.0 ; p=0.013\right)$, as well as a delay-bydistraction interaction $\left(F_{(2,16)}=3.65 ; p=0.05\right)$. Post hoc paired $t$ tests (two-tailed, mean, SEM) confirmed significantly increased memory performance when a $1 \mathrm{~s}$ distractor was presented during the $45 \mathrm{~s}$ delay period $(78.89 \pm 3.31 \%)$ compared with when no distractor was presented $\left(66.11 \pm 2.86 \%, t_{(8)}=-3.944, p=\right.$ 0.004; Fig. 4A), which was of similar magnitude to that found for BHS (Fig. 3). Conversely, $2 \times 3$ repeated-measures ANOVAs with delay length $(5,20,45 \mathrm{~s})$ and distraction (with and without) as the within-subjects factors in the high-performing NC3 group showed no main effects (all $p s>0.25$ ) and no interaction $\left(F_{(2,20)}=1.085 ; p=0.36\right)$. In addition, post hoc paired $t$ tests (two-tailed) did not show any memory improvement with distraction (all $p s>0.40$; Fig. $4 B$ ).

\section{Phase-coupling analysis}

To test our hypothesis that the presentation of task-unrelated stimuli during the delay periods disrupts maintenance, we analyzed interregional theta phase coupling (at $6 \mathrm{~Hz}$ during the delay period) of 10 healthy participants (NC1) from Study 1. Similar to Study 2, the MEG data were split into two groups based on the median performance of the high-load condition without distraction (median value $=81 \%$ accuracy): low performers $(\leq 80 \%$; $n=5$ participants; cumulative $50 \%$, mean age $26.8 \pm 7.5$ years, 4 females) and high performers ( $>80 \%: n=5$ participants cumulative $50 \%$, mean age $22.8 \pm 2.8$ years, 4 females). A $2 \times 2$ repeated-measures ANOVA with load (1 and 5 items) and distraction (distractor, no distractor) as the within-subjects factor and performance group (high and low performers) as the between group factors confirmed a significant distraction by group $\left(F_{(1,8)}=5.87 ; p=0.042\right)$ and a significant load-by-distractionby-group interaction $\left(F_{(1,8)}=6.80 ; p=0.031\right)$ for the relevant conditions used for subsequent MEG analysis. Furthermore, follow-up one-way ANOVA comparisons (mean, SEM) showed a significantly lower DMS performance for the 5-item load without distraction for the low performance group $\left(74.6 \pm 1.75 \%, F_{(1,8)}=\right.$ 29.27, $p=0.001$ ) compared with the high performance group $(86.6 \pm 1.36 \%)$, and this group difference was attenuated in the 5-item load distractor present condition (low performers: $79.4 \pm$ $3.8 \%$, high performers: $\left.85.5 \pm 8.8 \%, F_{(1,8)}=1.38, p=0.27\right)$.

Within-group serial-measures $t$ tests $(p<0.05$ over a time period of at least 2 theta cycles) on bifrontal sensor groups of the low performance group revealed significantly increased theta coupling for 5 -item load vs 1 -item load (Fig. 5A). This elevated bifrontal coupling was evident soon after stimulus offset and steadily increased across the delay period. In contrast, high performers had a similar trend for increased bifrontal coupling soon after stimulus offset (5-item load vs 1-item load), however, this enhancement diminished over the course of the delay (Fig. 5B). 
Between-group $t$ test comparisons of the 5 -item load condition (independent samples $t$ test, same threshold as above) on similar sensor groups showed that theta coupling decreased across the delay in the high-performing group, whereas it maintained this steady theta-coupled enhancement in the low-performing group (Fig. $5 C)$.

Within-group serial-measures $t$ tests ( $p<0.05$ threshold) contrasting 5-item load with and without delay distraction in the low performers group showed significant decreases of bifrontal coupling after the onset of distractor presentation $(\sim 2000 \mathrm{~ms})$ compared with the increased coupling in the absence of a distractor (Fig. 6A). In contrast, the 5-item load condition of the high-performing group resulted in no significant thetacoupling differences for delays with and without a distractor (Fig. 6B).

\section{Analysis of potential ocular artifacts in the MEG data}

A separate post hoc analysis was conducted to determine whether potential ocular artifacts (lateral eye movements or blinks) may have contributed to the primary phase-coupling analysis during the delay period. To this end, MEG data were preprocessed using Fieldtrip open source software (Oostenveld et al., 2011). Data were epoched (same as phase-coupling analysis above), down-sampled to $140 \mathrm{~Hz}$, and then band-pass filtered to $0.1-35 \mathrm{~Hz}$. A $200 \mathrm{~ms}$ prestimulus baseline correction was applied and then an independent component analysis (ICA) was conducted (separately for each condition per participant). ICA components containing vertical (blinks) and lateral eye movements were identified separately from the time window in which participants were instructed to blink (i.e., the intertrial interval just after probe; please see Material and Methods). Eye movements typically generate abrupt amplitude jumps in the frontal sensors and are easily identified with ICA in this manner (Mennes et al., 2010; Fatima et al., 2013). Using this approach, vertical and lateral eye movements can be identified separately by their characteristic sensor topography and signal profile (Joyce et al., 2004; Mognon et al., 2011; Tangermann et al., 2012; Fatima et al., 2013) due to the differences in signal propagation (Tangermann et al., 2012) from these two distinct forms of ocular muscle movements (Joyce et al., 2004). The number of eye movements (both vertical and lateral separately) during the delay period time windows (occurring just before probe) were then summed for each participant per condition.

One-way ANOVAs were then conducted for the mean number of vertical eye movements (blinks) identified during the delay
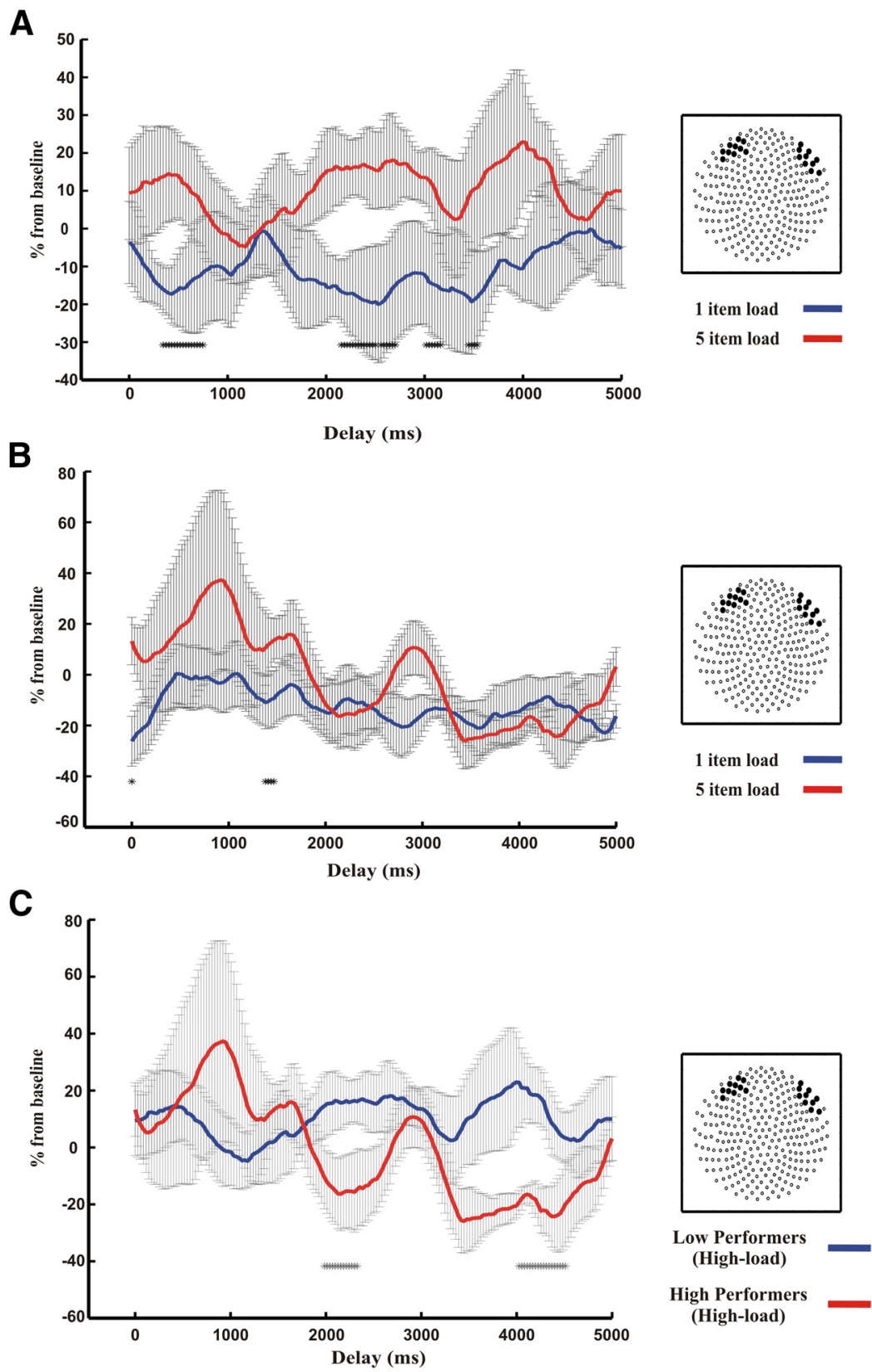

Figure 5. Bifrontal theta-band frequency synchrony in the high and low delayed match to sample (DMS) memory load conditions from Study 1. Serial-measures $t$ test comparisons of $6 \mathrm{~Hz}$ phase coupling on selected sensor groups (right, insets) for low DMS memory load (1-item load in blue) versus high DMS memory load (5-item load in red) delayed memory recognition conditions of normal controls (NC1) in Study 1 (threshold of $p<0.05$ per time point if present continuously over three successive theta cycles indicated by markings on $x$-axis). Error bars indicate SEM. $A$, In the low performers, bifrontal theta-coupling enhancement for the 5-item load condition continues throughout the memory delay compared with the 1-item load. However, in the high performers $(\boldsymbol{B})$, bifrontal theta coupling is enhanced in the early portion of the delay period for 5 -item load versus 1 -item load and then diminishes over the course of the delay. $\boldsymbol{C}$, Between-group comparisons of theta synchrony across a $5 \mathrm{~s}$ delay period of low performers (blue) versus high performers (red). Bifrontal theta coupling of the high performance group clearly declines over the course of the delay period, whereas in low performers, bifrontal theta coupling persists across the entire delay.

periods for each corresponding phase-coupling analysis comparison (Figs. 5, 6). Since the critical delay period time windows for the phase-coupling analysis were during the presentation of a face stimulus (1.5-3.5 s) in the distractor present conditions and during the latter portion of the delay period $(3.5-5 \mathrm{~s})$ for both the distractor present and no distractor conditions, the ICA results were also further subdivided in this manner for subsequent analysis. Therefore, the time windows over the entire delay were as 


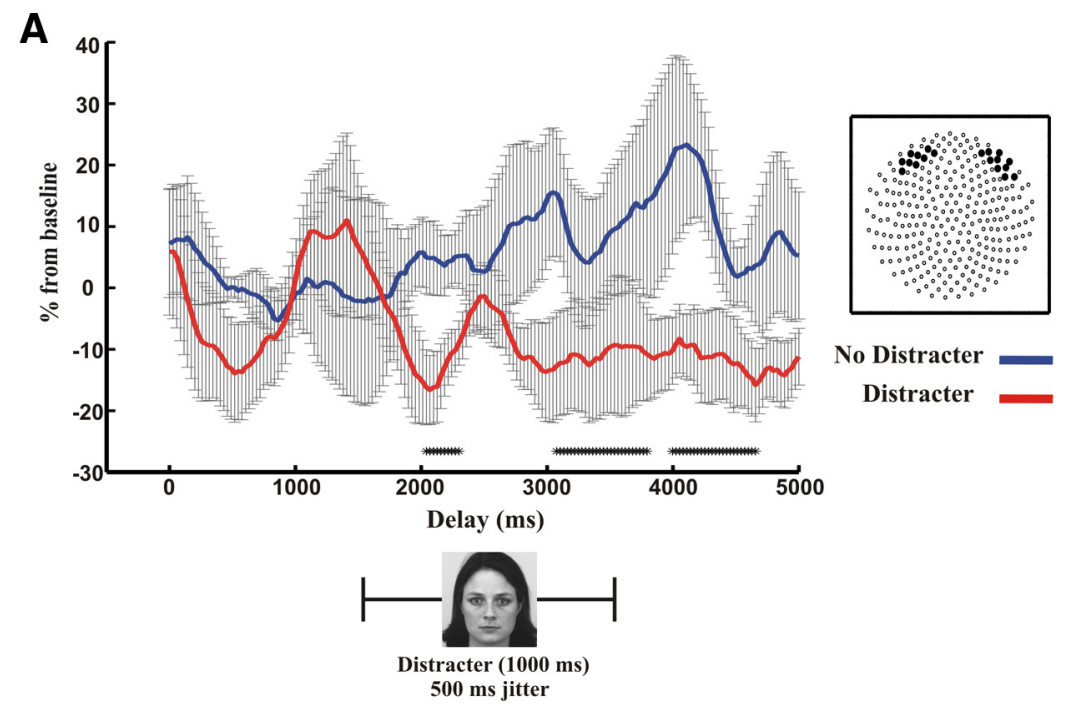

B
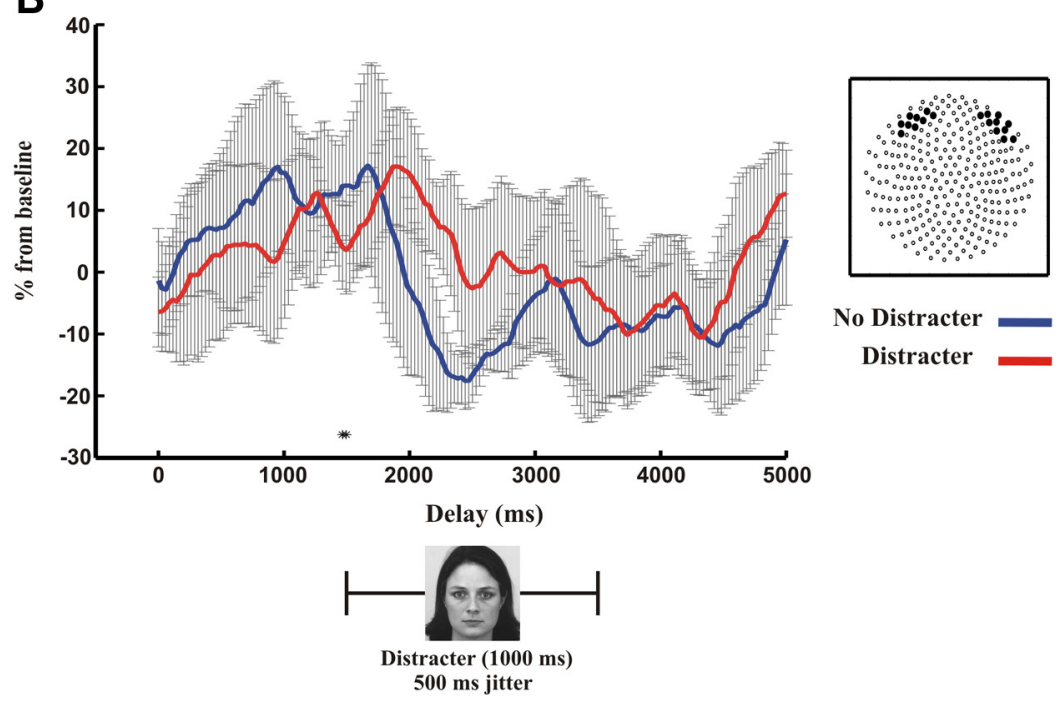

Figure 6. Bifrontal theta-band frequency synchrony of the high delayed match to sample (DMS) memory load (5 items) conditions with and without distraction for normal controls (NC1) in Study 1. Serial measures $t$ test comparisons of $6 \mathrm{~Hz}$ phase coupling (threshold of $p<0.05$ per time point if present continuously over three successive theta cycles indicated by markings on $x$-axis) on bifrontal sensor groups (right, insets). Error bars indicate SEM. $A$, Comparisons of theta synchrony across a 5 s delay period of low performers demonstrate that bifrontal theta coupling is reduced with distractor presentation (red) compared to without distractor presentation (blue). $\boldsymbol{B}$, In comparison, distractor presence (red) in the high performance group show no differences between bifrontal theta coupling compared with the no distraction condition (blue) during the delay period.

Table 3. Vertical eye movements

\begin{tabular}{llllrl}
\hline Group & Condition & $\begin{array}{l}\text { Entire delay } \\
(0-5.0 \mathrm{~s})\end{array}$ & $\begin{array}{l}\text { Start of delay } \\
(0-1.5 \mathrm{~s})\end{array}$ & \multicolumn{1}{c}{$\begin{array}{l}\text { Mid delay } \\
(1.5-3.5 \mathrm{~s})\end{array}$} & $\begin{array}{l}\text { End of delay } \\
(3.5-5 \mathrm{~s})\end{array}$ \\
\hline Low performers & 1-item & $30.2 \pm 9.7$ & $15.6 \pm 5.2$ & $7.8 \pm 1.9$ & $6.8 \pm 4.0$ \\
Low performers & 5-item & $38.6 \pm 10.6$ & $20.4 \pm 5.6$ & $10.4 \pm 4.5$ & $7.8 \pm 3.3$ \\
Low performers & 5-item (D) & $40.8 \pm 13.1$ & $21.6 \pm 6.3$ & $10.6 \pm 3.8$ & $8.6 \pm 4.0$ \\
High performers & 1-item & $26.6 \pm 9.1$ & $14.8 \pm 6.8$ & $7.4 \pm 3.0$ & $4.4 \pm 2.4$ \\
High performers & 5-item & $35.2 \pm 5.4$ & $20.6 \pm 3.2$ & $10.4 \pm 2.9$ & $6.0 \pm 1.9$ \\
High performers & 5-item (D) & $41.4 \pm 9.2$ & $20.4 \pm 4.5$ & $12.6 \pm 4.9$ & $8.4 \pm 2.8$ \\
\hline
\end{tabular}

The mean number of vertical eye movements (blinks) identified with independent component analysis during MEG recordings of DMS memory delays in NC1 (Study 1). There were no statistical differences in identified eye movements between memory load (1-item or 5 -item) or distractor present (D) conditions and also, no differences between high or low memory performance groups (all $p>0.11$ ); confirming that statistical comparisons of bifrontal theta coupling in NC1 were not notably influenced by eye movements during the MEG recordings.

follows: $0-5.0 \mathrm{~s}$ (beginning of delay): $0-1.5 \mathrm{~s}$ (middle of delay): 1.5-3.5 s (end of delay): 3.5-5 s; results summarized in Table 3). A one-way ANOVA of the 1-item load and 5-item load conditions in the low performers (same comparison as Fig. 5A) con- firmed that there were no differences in the number of identified vertical eye movements between conditions for any of the delay period time windows (all $p s>$ $0.20)$ and likewise for the high performers (same comparison as Fig. $5 B$; all $p s>$ $0.11)$.

A one-way ANOVA comparing the 5-item load conditions between high and low performers (same comparison as Fig. $5 C$ ) also confirmed that there were no differences between identified vertical eye movements during any delay period time window (all $p s>0.32$ ). In addition, oneway ANOVAs between the 5-item load without distraction and the 5-item load with distraction conditions confirmed that there were no differences in the number of identified vertical eye movements between conditions for the low performers (all ps > 0.74) or for the high performers (all ps $>0.15$ ) during any of the delay period time windows (same comparisons as Fig. $6 A, B$, respectively). The number of identified lateral eye movements during the delay period was considerably sparse (low performers $1.6 \pm 1.6$ and high performers $1.7 \pm 1.4)$ and therefore further statistical comparisons were not necessary.

A time-locked analysis was also calculated creating a time-series of event-related fields (ERFs) across the entire epoch and then grand averaged separately for each condition per performance group. The topographic distribution of the ERFs were then plotted for the same frontal sensor groups that demonstrated significant bifrontal theta coupling in the prior phase-coupling analysis during the critical delay period time window (1.5-5 s) and also the time window when participants were instructed to blink (intertrial interval used to identify ICA components containing eye movements; Fig. 7). Please note that the averaged ERF topographies during the intertrial intervals clearly demonstrate the typical bifrontal distribution created by eye blink artifacts (Mennes et al., 2010; Joyce et al., 2004; Mognon et al., 2011; Fatima et al., 2013;). In contrast, the ERF topographies during the critical delay period time window have a radically different distribution than eye blinks for all relevant conditions of comparison (Fig. 7). Therefore, the averaged MEG signal recorded on these sensor groups during the delay period was not notably contaminated by ocular movements during this time window and, more importantly, there were no differences in the number of ICA-identified eye movements for all conditions of comparison used for the phase-coupling analysis.

Although ICA is often used to remove components with eye movements for subsequent ERF analysis or other averaged time series analysis techniques (Mennes et al., 2010; Fatima et al., 2013), this was not a valid option for our current phase-coupling analysis due to the potential distortion of phase relationships with sensor location known to occur from extracting ICA com- 
A
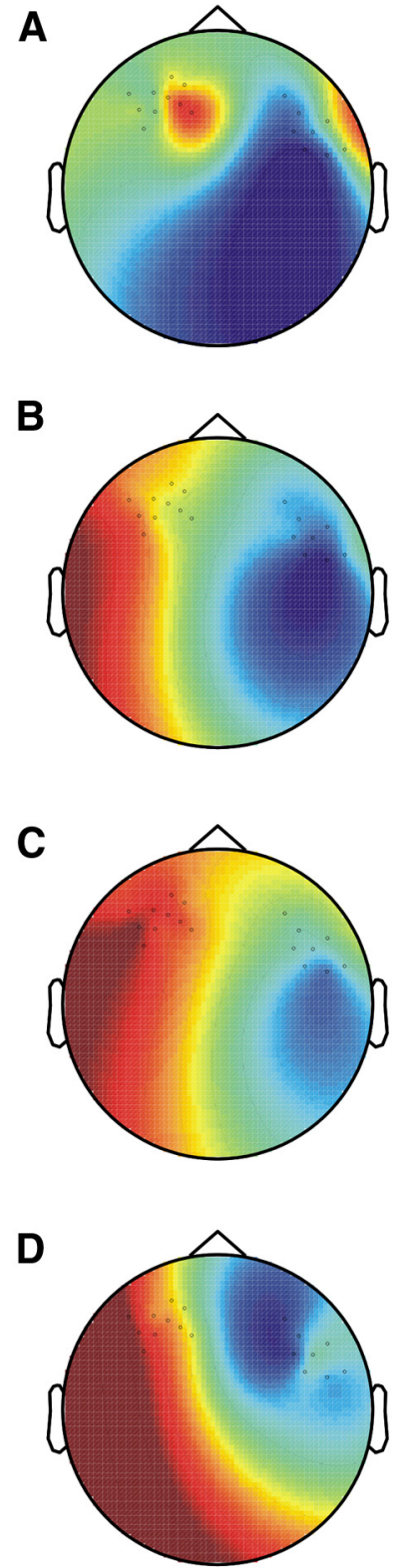

Delay Period
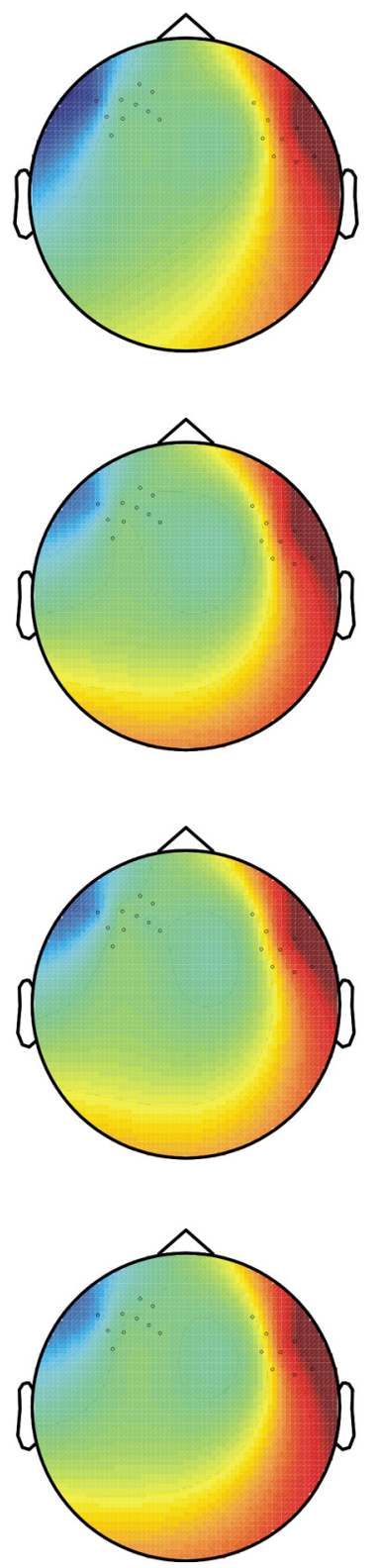

Inter-trial-interval

Figure 7. Topographic distribution of event-related fields (ERFs) for the critical time window of analysis during the delayed match to sample (DMS) delay period (left) and the intertrial interval when participants were instructed to blink (right) on the same bifrontal sensors as in the primary phase-coupling analysis in NC1 (Study 1 ) for all relevant conditions of comparison $(\boldsymbol{A}, 1$-item load in the low performers; $\boldsymbol{B}, 5$-item load in the low performers; $\boldsymbol{C}, 5$-item load with distraction in the low performers; and $\boldsymbol{D}, 5$-item load in the high performers). The ERF topographies during the intertrial intervals clearly demonstrate the typical bifrontal distribution created by eye blink artifacts (right). In contrast, the critical DMS delay period time windows used for analysis (left) have a radically different distribution, demonstrating that ocular movements were not notably contaminating DMS delay period MEG recordings during any of the primary conditions of comparison used for the phase-coupling analysis.

ponents (Wallstrom et al., 2004; Castellanos and Makarov, 2006; Kierkels et al., 2006; Thatcher, 2012). However, the ERF results demonstrated that the average signal within these sensor groups was not notably contaminated by eye movements (Fig. 7); and furthermore, the number of ICA-identified eye movements (Table 3 ) could not contribute to the statistical differences found in the primary phase-coupling analysis (Figs. 5, 6).

\section{Discussion}

Although distraction is typically known to impair memory, particularly in patients with memory dysfunction, here we report a clinically and theoretically relevant exception. We describe a beneficial effect of distractor presentation during memory rehearsal in patients with pronounced memory impairment after a bilateral lesion of the hippocampus (BHS), a brain area associated with amnesia. Furthermore, this distractor presentation elevated the memory performance of BHS patients to the level of a significantly younger and relatively better educated group of healthy controls (NC1). We also determined that differences in age and education (NC2) cannot account for the impairments found in BHS patients and that the beneficial effects of distractor presentation in the BHS group cannot be explained in terms of disease status or medication (TLE). Furthermore, we also establish a similar beneficial effect of distraction in healthy controls (NC3) with low memory performance.

MEG recordings of oscillatory brain activity in $\mathrm{NC1}$ participants provide a physiological account for these findings. Throughout the delay period, bifrontal MEG sensors have shown enhanced synchrony in the theta frequency range (Cashdollar et al., 2009) in the high-load compared with the low-load condition. Neural synchrony within the theta band is a plausible physiological mechanism mediating active maintenance (Jones and Wilson, 2005; Fuentemilla et al., 2010), or in other words, working memory rehearsal (Fuentemilla et al., 2010). A performance-based median split of the NC1 group revealed that this synchrony pattern was robust throughout the entire delay period in the low-performing, but not the highperforming, individuals (Fig. 5). The MEG results therefore suggest that ongoing working memory rehearsal (Jones and Wilson, 2005; Fuentemilla et al., 2010) is associated with low levels of performance when memory load is high. Furthermore, the bilateral frontal theta synchrony in low performers was markedly reduced immediately after distractor presentation (Fig. 6A), suggesting that distractor presentation disrupted rehearsal. In contrast, distractors had no effect on bifrontal theta synchronization in the high performance group (Fig. 6B).

These physiological observations indicate that not all healthy individuals use an active maintenance strategy when the demand on maintenance is high (5 items). Interindividual variability in the extent to which individuals afford active maintenance when load is high could explain why distractor-related memory enhancement in BHS patients is slightly less robust with high rather than with moderate levels of load (Fig. 3C). In fact, this difference between patients and controls is very meaningful because there is well founded evidence that patients with hippocampal lesions have working memory problems (Hannula et al., 2006; Cashdollar et al., 2009) and it is quite feasible that working memory capacity is lower with bilateral hippocampal lesions. It is important to note that this distraction-related improvement at higher memory loads is evident in every individual BHS patient in Study 1 (Table 2).

It seems likely that this improvement has not been characterized in previous studies (Postle et al., 2005; Clapp et al., 2010) potentially due to the already optimal memory performance of participants when presented with unrelated distractors during memory delays. Using identical stimuli and delay interval ( $5 \mathrm{~s})$ as presented here, we have previously demonstrated that distraction leads to impaired performance in the high-load condition when distracting stimuli belong to the same category as those currently held in memory and are task related (Cashdollar et al., 2009). It is conceivable that stimuli that belong to the same category have 
this negative effect because they lead to a further increase in memory load.

Our observation that memory performance can be improved by interrupting rehearsal raises the question of what memory signal is used instead. The most parsimonious explanation is a memory signal that enables familiarity-based recognition of a match between the probe stimulus and the stimulus in memory. It is well known that familiarity-based recognition of a memory match can be supported by brain regions adjacent to the hippocampus, the rhinal and parahippocampal cortex (Eichenbaum et al., 2007). Furthermore, lesions to the rhinal and parahippocampal regions in nonhuman primates impair recognition memory in tasks similar to those used here (Guderian et al., 2011). Lower performance may then result from a "faulty" working memory representation and its interference with rhinal or parahippocampal familiarity signals. In fact, physiological studies on persistent spiking, a form of active maintenance (Compte et al., 2000), show that random drifts in network activity lead to a loss in representational precision (tuning) over time (Compte et al., 2000). The observation that patients with bilateral hippocampal injury are impaired at much shorter delays (5s) than are healthy adults (45s) indicates that the hippocampus may help reduce such drifts thereby delaying the emergence of faulty representations under active maintenance. Although theta synchrony and persistent spiking are likely to be different mechanisms of active maintenance (Mehta, 2005), it is conceivable that similar drifts in representational precision occur in both.

The performance improvement with distractor presentation then can be explained by the interruption of the emerging faulty working memory representation and the reliance on rhinal or parahippocampal familiarity signals instead. Studies in patients with hippocampal injury have demonstrated relatively preserved familiarity-based recognition memory for lists of multiple items (Yonelinas et al., 2002). Such familiarity-based recognition would not be possible if memory for a given item were disrupted (in a distractor-like fashion) by the presentation of the next items in the list, suggesting that the familiarity signal may have a level of resilience to distraction.

Whether continual working memory rehearsal aids long-term recognition memory has been of long-standing theoretical interest (Atkinson and Shiffrin, 1971; Baddeley, 1998, 2003; Hasselmo and Stern, 2006; Axmacher et al., 2007). Recent evidence has indicated that short-term persistence of information within working memory can enhance long-term retention of the same information (Schon et al., 2004; Ranganath et al., 2005; Khader et al., 2007). Our results suggest that in cases in which visual working memory is challenging (due to hippocampal lesions or individual variation in the healthy population), ongoing rehearsal does not necessarily improve the quality of recognition memory signals, but may in fact degrade them.

Paradoxical effects of cognitive improvements in patients with brain lesions, as demonstrated here, are quite rare (but see Warrington and Davidoff, 2000; Reverberi et al., 2005). However, they are theoretically interesting because they circumvent the typical ambiguities associated with mapping a behavioral impairment onto a brain lesion. A behavioral impairment could be a result of a disruption along any chain of cascading neural events downstream of the lesion of interest. Whereas in cases of paradoxical improvement, as observed here, a potential contamination by downstream dysfunction can be ruled out because this would lead to a deficit rather than an improvement in behavior (for detailed discussion, see Reverberi et al., 2005). Hippocampal injury has been known to impair retention when short-term memory load is high (Shrager et al., 2008; Jeneson et al., 2011; Jeneson et al., 2012), yet our results highlight circumstances in which disrupting this process can improve recognition memory by allowing an alternate (and potentially competing) memory process to persist that does not rely on the hippocampus. The therapeutic implication of these interactions between memory processes for patients with hippocampal injury is counterintuitive at first; rehearsal of information may paradoxically exacerbate their memory impairment and interfere with optimal usage of their remaining memory capacities. Our data demonstrate conditions that can elevate memory performance in these patients to the level of young healthy adults.

\section{References}

Atkinson RC, Shiffrin RM (1971) The control of short-term memory. Sci Am 225:82-90. CrossRef Medline

Axmacher N, Mormann F, Fernández G, Cohen MX, Elger CE, Fell J (2007) Sustained neural activity patterns during working memory in the human medial temporal lobe. J Neurosci 27:7807-7816. CrossRef Medline

Baddeley A (1998) Recent developments in working memory. Curr Opin Neurobiol 8:234-238. CrossRef Medline

Baddeley A (2003) Working memory and language: an overview. J Commun Disord 36:189-208. CrossRef Medline

Cashdollar N, Malecki U, Rugg-Gunn FJ, Duncan JS, Lavie N, Düzel E (2009) Hippocampal dependent and independent theta networks in human working memory. Proc Natl Acad Sci U S A 106:20493-20498. CrossRef Medline

Castellanos NP, Makarov VA (2006) Recovering EEG brain signals: artifact suppression with wavelet enhanced independent component analysis. J Neurosci Methods 158:300-312. CrossRef Medline

Chao LL, Knight RT (1995) Human prefrontal lesions increase distractibility to irrelevant sensory inputs. Neuroreport 6:1605-1610. CrossRef Medline

Chao LL, Knight RT (1998) Contribution of human prefrontal cortex to delay performance. J Cogn Neurosci 10:167-177. CrossRef Medline

Clapp WC, Gazzaley A (2012) Distinct mechanisms for the impact of distraction and interruption on working memory in aging. Neurobiol Aging 33:134-148. CrossRef Medline

Clapp WC, Rubens MT, Gazzaley A (2010) Mechanisms of working memory disruption by external interference. Cereb Cortex 20:859-872. CrossRef Medline

Compte A, Brunel N, Goldman-Rakic PS, Wang XJ (2000) Synaptic mechanisms and network dynamics underlying spatial working memory in a cortical network model. Cereb Cortex 10:910-923. CrossRef Medline

Eichenbaum H, Yonelinas AP, Ranganath C (2007) The medial temporal lobe and recognition memory. Annu Rev Neurosci 30:123-152. CrossRef Medline

Fatima Z, Quraan MA, Kovacevic N, McIntosh AR (2013) ICA-based artifact correction improves spatial localization of adaptive spatial filters in MEG. Neuroimage 78:284-294. CrossRef Medline

Fuentemilla L, Penny WD, Cashdollar N, Bunzeck N, Düzel E (2010) Thetacoupled periodic replay in working memory. Curr Biol 20:606-612. CrossRef Medline

Guderian S, Düzel E (2005) Induced theta oscillations mediate large-scale synchrony with mediotemporal areas during recollection in humans. Hippocampus 15:901-912. CrossRef Medline

Guderian S, Brigham D, Mishkin M (2011) Two processes support visual recognition memory in rhesus monkeys. Proc Natl Acad Sci U S A 108: 19425-19430. CrossRef Medline

Hannula DE, Tranel D, Cohen NJ (2006) The long and the short of it: relational impairment in amnesia, even at short lags. J Neurosci 26:83528359. CrossRef Medline

Hasselmo ME, Stern CE (2006) Mechanisms underlying working memory for novel information. Trends Cogn Sci 10:487-493. CrossRef Medline

Jeneson A, Mauldin KN, Hopkins RO, Squire LR (2011) The role of the hippocampus in retaining relational information across short delays: the importance of memory load. Learn Mem 18:301-305. CrossRef Medline

Jeneson A, Wixted JT, Hopkins RO, Squire LR (2012) Visual working memory capacity and the medial temporal lobe. J Neurosci 32:3584-3589. CrossRef Medline 
Jones MW, Wilson MA (2005) Theta rhythms coordinate hippocampalprefrontal interactions in a spatial memory task. PLoS Biol 3:e402. CrossRef Medline

Joyce CA, Gorodnitsky IF, Kutas M (2004) Automatic removal of eye movement and blink artifacts from EEG data using blind component separation. Psychophysiology 41:313-325. Medline

Khader P, Ranganath C, Seemüller A, Rösler F (2007) Working memory maintenance contributes to long-term memory formation: evidence from slow event-related brain potentials. Cogn Affect Behav Neurosci 7:212224. Medline

Kierkels J, van Boxtel G, Vogten LL (2006) A model-based objective evaluation of eye movement correction in EEG recordings. IEEE Trans Biomed Eng 53:246-253. CrossRef Medline

Lachaux JP, Rodriguez E, Martinerie J, Varela FJ (1999) Measuring phase synchrony in brain signals. Hum Brain Mapp 8:194-208. CrossRef Medline

McGeoch JA, McDonald WT (1931) Meaningful relation and retroactive inhibition. Am J Psychol 44:579-588.

Mehta MR (2005) Role of rhythms in facilitating short-term memory. Neuron 45:7-9. CrossRef Medline

Mennes M, Wouters H, Vanrumste B, Lagae L, Stiers P (2010) Validation of ICA as a tool to remove eye movement artifacts from EEG/ERP. Psychophysiology 47:1142-1150. CrossRef Medline

Mognon A, Jovicich J, Bruzzone L, Buiatti M (2011) ADJUST: an automatic EEG artifact detector based on the joint use of spatial and temporal features. Psychophysiology 48:229-240. CrossRef

Oostenveld R, Fries P, Maris E, Schoffelen JM (2011) Field Trip: Open source software for advanced analysis of MEG, EEG, and invasive electrophysiological data. Comput Intell Neurosci 2011:156869. CrossRef Medline

Postle BR, Desposito M, Corkin S (2005) Effects of verbal and nonverbal interference on spatial and object visual working memory. Mem Cognit 33:203-212. CrossRef Medline

Ranganath C, Cohen MX, Brozinsky CJ (2005) Working memory maintenance contributes to long-term memory formation: neural and behavioral evidence. J Cogn Neurosci 17:994-1010. CrossRef Medline

Reverberi C, Toraldo A, D’Agostini S, Skrap M (2005) Better without (lateral) frontal cortex? Insight problems solved by frontal patients. Brain 128:2882-2890. CrossRef Medline
Saksida LM, Bussey TJ (2010) The representational-hierarchical view of amnesia: translation from animal to human. Neuropsychologia 48:2370 2384. CrossRef Medline

Schon K, Hasselmo ME, Lopresti ML, Tricarico MD, Stern CE (2004) Persistence of parahippocampal representation in the absence of stimulus input enhances long-term encoding: a functional magnetic resonance imaging study of subsequent memory after a delayed match-to-sample task. J Neurosci 24:11088-11097. CrossRef Medline

Shrager Y, Levy DA, Hopkins RO, Squire LR (2008) Working memory and the organization of brain systems. J Neurosci 28:4818-4822. CrossRef Medline

Squire LR, Stark CE, Clark RE (2004) The medial temporal lobe. Annu Rev Neurosci 27:279-306. CrossRef Medline

Tangermann M, Müller KR, Aertsen A, Birbaumer N, Braun C, Brunner C, Leeb R, Mehring C, Miller KJ, Müller-Putz GR, Nolte G, Pfurtscheller G, Preissl H, Schalk G, Schlögl A, Vidaurre C, Waldert S, Blankertz B (2012) Review of the BCI Competition IV. Front Neurosci 6:55. CrossRef Medline

Thatcher RW (2012) Coherence, Phase Differences, Phase Shift, and Phase Lock in EEG/ERP Analyses. Dev Neuropsychol 37:476-496. CrossRef Medline

Vargha-Khadem F, Gadian DG, Watkins KE, Connelly A, Van Paesschen W, Mishkin M (1997) Differential effects of early hippocampal pathology on episodic and semantic memory. Science 277:376-380. CrossRef Medline

Wallstrom GL, Kass RE, Miller A, Cohn JF, Fox NA (2004) Automatic correction of ocular artifacts in the EEG: a comparison of regression-based and component-based methods. Int J Psychophysiol 53:105-119. CrossRef Medline

Warrington EK, Davidoff J (2000) Failure at object identification improves mirror image matching. Neuropsychologia 38:1229-1234. CrossRef Medline

Yonelinas AP, Kroll NE, Quamme JR, Lazzara MM, Sauvé MJ, Widaman KF, Knight RT (2002) Effects of extensive temporal lobe damage or mild hypoxia on recollection and familiarity. Nat Neurosci 5:1236-1241. Medline 